

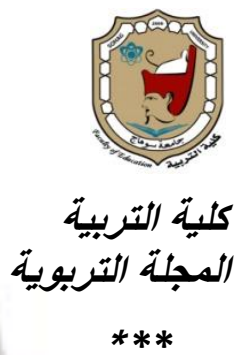

"Les compétences informationnelles nécessaires à la

formation contenue et le degré de leur présence chez les enseignants actuels du français langue étrangère

Dr. Doaa Mohammad Hamdan Ahmed Maître de conférences en didactique du FLÉ, Faculté de l'éducation , Université de Sohag

المجلة التربوية ـ العدد الخامس والستوز ـ سبتمبر 19+rم م

Print:(ISSN 1687-2649) Online:(ISSN 2536-9091) 
Résumé : Dans notre société du savoir numérique, la maitrise des compétences informationnelles devient une nécessité qui permet aux individus d'être compétitifs et favorise leur intégration sociale (Potvin, 2013). La présente étude est une étude descriptive qui s'inscrit dans ce domaine de la recherche: les compétences informationnelles. Elle vise principalement à identifier les compétences professionnelles sur le plan informationnel et de vérifier le degré de leur maîtrise auprès des enseignants actuels du français langue étrangère. Pour ce faire, une grille a été proposée pour déterminer les compétences informationnelles visées; un questionnaire a été élaboré et présenté à un échantillon des 45 enseignants du FLÉ ainsi qu'un entretien semi-dirigé a été individuellement effectué avec certains d'eux (15 enseignants). Les résultats de l'étude montrent cinq types des compétences informationnelles: des compétences informationnelles générales, des compétences d'un besoin informationnel, des compétences de la recherche, des compétences de l'évaluation et des compétences de l'utilisation de l'information sur le Web. Les résultats indiquent également que les enseignants participants sont compétents dans la précision de leur besoin informationnel; ils ont un niveau moyen dans les compétences informationnelles générales et dans celles de la recherche de l'information sur le Web, alors que leur niveau est encore débutant dans celles qui sont relatives à l'évaluation et à l'utilisation de l'information sur le Web. L'étude recommande d'intégrer ces compétences informationnelles dans les programmes de la formation académique et professionnelle de futurs enseignants du FLÉ.

Mots-clés : Les technologies de l'information et de la communication, les compétences informationnelles, la formation contenue, les enseignants actuels, le français langue étrangère

Summary: In our digital knowledge society, mastery of information skills becomes a necessity not only because it allows individuals to be competitive but also it promotes their social integration (Potvin, 2013). Information skills are the theme of this descriptive study that aimed primarily at identifying information-related professional skills. The study also examined the degree of their competency among mentors teaching French as a foreign language. To achieve these goals, the following plans were done: a grid was proposed to determine information skills; a questionnaire was developed and presented to a sample of 45 mentors teaching French as a foreign language and a semi-structured interview was individually performed with some of these mentors (15 teachers). The results show five types of information skills including general information skills, skills of determining the need for specific information, research skills, assessment skills and skills of using information on the Web. The mentors (teachers) participating in the study were competent in skills of determining the need for a specific information. Their level of competence was average in general information skills and research skills. Alternatively, their level of competence in the remaining skills (assessment skills and skills of using information on the Web) was below average (weak). To conclude, the current study recommends the inclusion of these information skills in the curriculum of the future academic and professional training designed for the mentors (teachers) teaching French as a foreign language.

Key-words: Information and Communication Technology, informational skills, continued training, current mentors, French as a foreign language 


\section{口املغص الدراسة}

إن إتقان المهارات المعلوماتية فى ظل مجتمع المعرفة الرقمية الذى نعيشه الأن أصبح و على الإندماج (Potvin, 2013)ضرورةً أساسية، إذ يساعد الأفراد على المنافسة . الاجتماعي

تندرج الاراسة الحالية تحت هذا المجال البحثي و هو مجال المهارات المعلوماتية، و تهدف هذه الاراسة الوصفية بشكل أساسي إلى التعرف على المهارات المهنية اللازم توافرها لاى المعلمين على المستوى المعلوماتي بشكل عام و مدى اتقان هذه المهارات لاى معلمي اللغة الفرنسية ممن هم أثناء الخدمة بوجه خاص. و لتحقيق أهداف الدراسة تم إعداد قائمة مقترحة للتعرف على المهارات المعلوماتية اللازم إتقانها لاى هؤلاء المعلمين، كما تم إعداد و توجيه استبيان لعينة مكونة من خمس و أريعين معلمًا للغة الفرنسية، كذلك تم عقد مقابلات فردية شبه منظمة مع خمسة عشر معلمًا منهم. وقد أشارت نتائج الدراسة إلى وجود خمسة مجالات أساسية من المهارات المعلوماتية اللازم توافرها لاى المعلمين و التي تتمثل في: المهارات المعلوماتية العامة، مهارات تحديد الحاجة إلى المعلومات الرقمية، مهارات البحث عن المعلومات الرقمية، مهارات تقويم المعلومات التى تم الحصول عليها و مهارات استخدام هذه المعلومات على الثبكة العنكبوتية. و فيما يخص مدى إتقان معلمي اللغة الفرنسية لهذه المهارات توصلت الدراسة إلى إتقان هؤلاء المعلمون لمهارات تحديد الحاجة إلى المعلومات الرقمية، وهم ذ ذوي مستوى متوسط فيما يتعلق بالمهارات المعلوماتية بشكل عام و مهارات البحث عن المعلومات الرقمية، بينما يتميزون بمستوى مبتدئ فى مهارات استخدام و تقويم المعلومات على الثبكة العنكبوتية. و توصي الاراسة بضرورة تضمين هذه المهارات فى برامج الإعداد الأكاديمي و المهني لمعلمي المستقبل في مجال تعليم اللغة الفرنسية. الكلعات المفتاهيلة تكنولوجيا المطلومات و الاتصال - المهارات المعلوماتية - الإعداد المهني المستمر - معلمي اللغة الفرنسية كلفة أجنبية 


\section{Introduction et problème de la recherche}

L'utilisation des technologies de l'information et de la communication (TIC) évolue constamment en prenant une place considérable dans le quotidien de chacun (Brich et Irvine, 2009; Teo, Chai, Hung et Lee, 2008). Cette utilisation a influencé de façon significative la manière de la vie sur le plan économique, social et éducatif (Dohn, 2009; Redecker, 2009). L'éducation est un des secteurs les plus influencés par l'usage pédagogique des TIC (Drent et Meelissen, 2008; Fox, 2007; Underwood et Dillon, 2011). En effet, la présence de ces technologies en contexte scolaire comporte à la fois certains avantages et défis qui devraient soulever de nouvelles questions à l'intention des acteurs de l'éducation (Karsenti et Collin, 2013). D'une part, l'utilisation des TIC conduit l'apprenant à la nécessité de la participation à son apprentissage, il s'agit qu'il doive apprendre comment apprendre. L'accent est ici mis sur l'acquisition de ce qu'on appelle les compétences informationnelles qui lui permettent d'être plus indépendant, plus autonome, d'être apte à apprendre tout au long de sa vie et de répondre aux défis du monde informationnel (Sacchanand, 2011). L'enseignant devient, d'autre part, le guide ou le moteur de l'enseignement/apprentissage qui doit aider ses élèves à confronter à la société du savoir qui les entoure, ce qui l'invite à la nécessité de maîtriser lui-même les compétences de l'information.

Les TIC, y compris les téléphones cellulaires, les ordinateurs portables, les blocs-notes, les tablettes, etc., ont offert de nombreux avatars aux apprenants, et ce, dès leur âge le plus jeune. L'intégration de ces appareils technologiques en éducation et leur application en salles de classe ont pris de plus en plus d'importance pour les apprenants durant leur scolarité (Dohn, 2009; Galanouli et Mcnair, 2001). Cette intégration se transpose également dans la formation des enseignants (Chai et Lim, 2011; Fox, 2007). Ces derniers ont actuellement recours à l'utilisation des TIC pour répondre à leurs intérêts et à leurs besoins éducatifs lors de la pratique de leur métier (Dumouchel, 2016). Dans le monde des TIC, comme le montre Loisy (2007), les enseignants doivent avoir deux types des compétences : des compétences professionnelles en général qui sont relatives à la pratique de leur travail pédagogique et des compétences professionnelles sur un plan informationnel qui leur permettent d'intégrer les TIC dans les situations éducatives. Dans le même sens, le ministère de l'éducation nationale, de l'enseignement supérieur et de la 
recherche (2013) distingue plusieurs compétences à maîtriser auprès des enseignants. Parmi celles-ci, on trouve l'intégration des TIC dans le processus de l'enseignement et le pouvoir d'aider les élèves à profiter des avantages des TIC dans leur scolarité.

Dans le contexte égyptien et plus précisément en enseignement du français langue étrangère (FLÉ), nombreuses études se sont intéressées aux TIC. Certaines études ont visé à développer les compétences de l'écrit (lecture/écriture) et de l'écoute chez les étudiants universitaires les futurs enseignants - à partir de l'intégration des TIC dans le processus de l'enseignement (Othman, 2008; Salem, 2009, 2010, 2012, 2013; Waguih, 2002). Pour ce faire, les chercheurs ont utilisé les sites Web, le multimédia, le cyber enquête, ce qui avait son influence positive sur le développement des compétences linguistiques visées en français. Quant à la question des compétences informationnelles, nombreuses sont les études menées (April et Beaudoin, 2006; Dumouchel, 2016; Giroux, Gagnon, Gremion et Heinzen, 2014), mais à la connaissance de la chercheuse, il existe un manque dans les études égyptiennes menées sur ce domaine en FLÉ, et ce, d'une part.

D'autre part, le ministère de l'éducation fait constamment des réformes dans le système de l'enseignement en présentant tout ce qui est nouveau dans le processus de l'enseignement/ apprentissage, soit des livres scolaires, des méthodes d'enseignement, des activités d'apprentissage, etc. Dans cette année académique 2018/2019, le ministère essaie d'appliquer un système bien nouveau en enseignement ainsi qu'en évaluation des performances attendues des apprenants égyptiens. L'utilisation des Tablettes numériques qui comportent les curricula et les vidéos éducatives, l'utilisation de l'internet à l'école et l'évaluation en ligne sont certains traits qui caractérisent ce système proposé. Le rôle de l'enseignant consiste alors à aider les élèves à apprendre comment apprendre par le moyen de ces appareils et non par comme un présentateur pour les informations et les curricula. Puisque les enseignants sont toujours la clé de l'usage des technologies en éducation en général et en salles de classe, en particulier (Walha et Peters, 2011).

L'importance de la maîtrise de l'information dans notre monde numérique, le manque des études égyptiennes menées sur les 
compétences nécessaires dans ce domaine et les changements ministériels récemment présentés invitent à consacrer l'étude actuelle à s'intéresser aux compétences informationnelles chez les enseignants actuels. L'étude vise donc à atteindre deux objectifs essentiels qui sont : identifier les compétences informationnelles qui sont nécessaires à maîtriser afin de réaliser la formation contenue des enseignants dans la société du savoir et d'examiner le degré de la maîtrise de ces compétences auprès des enseignants actuels du FLÉ.

\section{Questions de l'étude}

L'étude essaie de répondre à ces deux questions soulevées :

1. Quelles sont les compétences informationnelles à maîtriser afin d'atteindre la formation contenue des enseignants?

2. Quel est le degré de la maîtrise des compétences informationnelles chez les enseignants actuels du FLÉ?

\section{Pertinence scientifique et pratique de l'étude}

L'étude actuelle traite des compétences fondamentales à la formation contenue des enseignants et qui sont relatives à l'univers de l'information numérique. Comme le montrent l'OCDE (2009) et l'UNESCO (2005), les compétences informationnelles sont utiles et nécessaires pour réussir dans les différentes sphères de la vie contemporaine. L'importance de ces compétences s'accroît auprès des enseignants actuels qui sont responsables d'exécuter les progressions ministérielles et de développer les compétences informationnelles chez leurs élèves. La question de cette étude est donc pertinente dans la vie personnelle, sociale et professionnelle des enseignants. Les résultats présentent une grille des compétences informationnelles que les enseignants - actuels et futurs - doivent avoir pour continuer dans la pratique de leur profession. Les résultats présentent également un portrait pour la présence de ces compétences chez un échantillon des enseignants en FLÉ qui sont en poste, ce qui leur permet d'examiner leur niveau actuel par rapport à ces compétences et de savoir quelles sont les compétences qu'ils doivent développer au futur.

\section{Cadre théorique}

Le cadre théorique présente un descriptif de plusieurs enjeux soulevés dans l'étude, ce qui permet de mieux comprendre les données et les résultats obtenus. Il commence par la conception de compétence, d'information, de compétences informationnelles sur le Web, ensuite, les 
Les compétences informationnelles nécessaires à la formation contenue $\ldots \ldots \ldots \ldots \ldots \ldots$

compétences informationnelles sur le Web que les futurs enseignants doivent maîtriser et les modèles proposés dans la formation informationnelle sur le Web et, enfin, les études menées sur ce domaine de recherche.

\subsection{La compétence, l'information et les compétences informationnelles}

La littéracie informationnelle, la culture informationnelle, la conscience informationnelle, l'alphabétisme numérique, la maîtrise de l'information et les compétences informationnelles sont des termes qui se posent vigoureusement dans tous les secteurs de la vie sociale et quotidienne des individus et des sociétés. Les compétences informationnelles sont le terme le plus usagé dans le domaine de l'éducation (Chevilotte, 2007), notamment dans le processus de l'enseignement/apprentissage. Les lignes qui suivent présentent la conception de compétence, d'information et de compétences informationnelles.

\subsubsection{La compétence}

La conception de la compétence indique les connaissances, les habilités et les dispositions qui permettent aux apprenants d'agir (Conseil de l'Europe, 2001). Elle se définit, selon Tardif (2001, p.22) comme un «savoir-agir complexe prenant appui sur la mobilisation et la combinaison efficaces d'une variété de ressources internes et externes à l'intérieur d'une famille de situation ». Elle est également un ensemble des aptitudes, des connaissances, des qualifications et des savoir-faire que l'individu peut utiliser afin d'accomplir une action (OCDE, 1996). Ces auteurs soulignent ainsi que la compétence est un ensemble des connaissances, des habilités et des aptitudes qui permettent à l'individu d'accomplir une ou plus d'une tâche. Dans le même sens, Dumouchel (2016) montre qu'en ce qui concerne la compétence, l'individu fait une activité en se mettant en action, en mettant en œuvre et en mobilisant des ressources. Ces ressources peuvent être personnelles et elles combinent des savoirs, des savoir-faire et des savoir-être (Jonnaert, Barrette, Boufrahi et Masciotra, 2004; Legendre, 2005). Selon ces derniers auteurs, les savoirs constituent les connaissances; les savoir-faire présentent les connaissances procédurales, les habilités et les capacités; alors que les savoir-être indiquent les attitudes. Jonnaert et al., (2004) 
confirment les définitions précédentes en montrant que la compétence signifie

« la mise en œuvre par une personne en situation, dans un contexte déterminé, d'un ensemble diversifié, mais coordonné de ressources; cette mise en œuvre repose sur le choix, la mobilisation et l'organisation de ces ressources et sur les actions pertinentes qu'elles permettent pour un traitement réussi de cette situation » (P. 674).

\subsubsection{L'information}

Le terme de l'information a reçu plusieurs définitions qui sont variées selon les contextes de la recherche (Bawden, 2001). Le dictionnaire de Robert (1995, p.605) indique que l'information signifie «donner une forme, une structure, transmettre des connaissances, des renseignements ». Alors que le dictionnaire de Petit Larousse (1995, p. 550) définit l'information comme «un renseignement obtenu de quelqu'un ou sur quelque chose, ou une nouvelle communiquée par une agence de presse, un journal, la radio, la télévision ». Pour leur part, Pomagni et Wild (1998, p. 92) voient l'information comme un «renseignement qui améliore notre connaissance sur un sujet quelconque ». Il existe ainsi une certaine relation entre l'information et la connaissance. Arsac (2003) montre que l'information est la forme portant la connaissance et mobilisant la pensée. L'apprenant interprète l'information et l'intègre dans son réseau de connaissances, ce qui signifie qu'il transforme les informations en connaissances (Basque, 2005). Mais, la maîtrise de cette information dépasse la conception de l'information et elle indique à «un ensemble d'activités, une méthodologie et une démarche plus aboutie et plus complète » (Chevillote, 2007, p. 18).

\subsubsection{Les compétences informationnelles sur le Web}

Le terme des compétences professionnelles sur le plan informationnel sur le Web a occupé de plus en plus d'importance notamment dans les dernières années. Plusieurs conceptions ont été présentées pour le définir. La Conférence des Recteurs et des Principaux des Universités du Québec définit les compétences informationnelles comme l'ensemble des aptitudes permettant aux individus de déterminer les moments où ils ont un besoin d'information et de trouver, d'évaluer et d'utiliser cette 
information (CREPUQ, 2005). Une définition identique a été introduite par Chevillotte (2005, p. 43) qui voit qu'elles sont un ensemble de compétences permettant de reconnaître l'existence d'un besoin d'information, d'identifier l'information adéquate, de la trouver, de l'évaluer et de l'exploiter en relation avec une situation donnée, dans une perspective de résolution de problème ». Pour sa part, Aillerie (2012) montre que les compétences informationnelles correspondent à un ensemble d'habiletés qui permettent d'identifier quelle information est nécessaire, de localiser, d'évaluer et d'utiliser les informations trouvées dans une démarche de résolution de problème qui aboutie à une communication de l'information abordée. Puren (2008, p.1-2) montre également que la compétence informationnelle comme une «capacité à agir sur et par l'information en tant qu'acteur social: agir sur l'information implique de l'identifier, la trouver, l'évaluer, l'organiser et au besoin en créer; agir par l'information implique de l'utiliser et de la communiquer ». Il apparaît qu'il existe un accord parmi les auteurs concernant la conception de ce type des compétences, puisqu'ils les définissent comme un ensemble des habilités ou des capacités qui se trouvent dans le cadre de quatre domaines liés à l'information sur le Web : préciser le besoin d'information, trouver l'information, évaluer l'information et utiliser cette information.

\subsection{Les compétences informationnelles sur le Web à maîtriser}

Dans la société du savoir, les compétences informationnelles sur le Web sont d'importance capitale, notamment en éducation (UNESCO, 2006), ce qui a invité plusieurs chercheurs (Gervais, 2004; Gervaiset Clément, 2005; Kaplan et Haenlein, 2010; Loisy, 2007; Pinte, 2005) à en aborder. De nombreux rapports, des documents et des études s'attachent également à chercher l'identification des composantes et des indicateurs reliés à la maîtrise de l'information chez les apprenants, notamment les étudiants universitaires ou les futurs enseignants.

Le ministère de l'éducation du Québec a officiellement présenté un référentiel de compétences professionnelles pour la profession enseignante. Ce document a comporté douze compétences qui doivent être développées dans le cadre du programme de la formation des maîtres québécois. La huitième compétence consiste à intégrer les technologies de l'information et des communications (TIC) aux fins de préparation et 
de pilotage d'activités d'enseignement-apprentissage, de gestion de l'enseignement et de développement professionnel (Martinet, Raymond et Gauthier, 2001). Cette compétence comprend six composantes qui sont les suivantes (Martinet et al., 2001; Villeneuve, Karsenti et Collin, 2013) : exercer un esprit critique et nuancé par rapport aux avantages et aux limites des TIC comme soutien à l'enseignement/apprentissage, évaluer le potentiel didactique des outils informationnels et des réseaux en relation avec le développement des compétences du programme de formation, communiquer à l'aide d'outils multimédias, utiliser les TIC pour rechercher, interpréter, communiquer de l'information et résoudre des problèmes, utiliser les TIC pour constituer des réseaux d'échange et de formation continue et aider les élèves à s'approprier les TIC, à les utiliser dans les activités d'apprentissage, à évaluer leur utilisation et à juger les données trouvées.

En outre, le programme du Fonds de développement académique du réseau (FODAR, 2004) a introduit certaines compétences informationnelles que l'étudiant universitaire doit avoir pour être efficace dans ses études ainsi que dans son travail à venir. Parmi ces compétences : la précision du besoin d'information, le bon choix du vocabulaire (mots-clés) pour faire une recherche, l'usage des sources pertinentes d'information, l'élaboration de stratégies de recherche, le repérage de l'information à partir de différents outils de recherche, la sélection et l'évaluation des résultats d'une recherche réalisée, l'obtention des informations et des documents demandés, l'intégration de l'information nouvelle aux connaissances déjà existantes, l'organisation et la gestion de l'information, la communication et l'utilisation de l'information obtenue et la citation des sources utilisées dans la recherche.

D'ailleurs, le ministère de l'éducation nationale, de l'enseignement supérieur et de la recherche (2013) précise certaines compétences que l'enseignant doit maîtriser, dont la compétence n.9 : intégrer les éléments de la culture numérique nécessaires à l'exercice de son métier. Dans ce sens, le ministère montre que l'enseignant doit aider ses élèves à s'approprier les outils et les usages numériques de manière critique et créative, et il doit participer à l'éducation des élèves à un usage responsable d'internet et utiliser les technologies de manière efficace pour échanger et se former. 
Pour sa part, Karsenti (2015) a identifié six compétences informationnelles essentielles que la formation des apprenants doit comprendre à l'heure du Web qui sont: rechercher de l'information, évaluer si l'information trouvée est fiable, organiser l'information trouvée, communiquer efficacement l'information trouvée, rester informé des sujets qui répondent à l'intérêt de l'enseignant et comprendre fonctionnement des moteurs de recherche.

Selon l'association des directeurs et personnels de direction des bibliothèques universitaires et de

la documentation (2012, p.28-29), il existe quatre compétences primordiales: identifier le besoin d'information, produire et communiquer, accéder aux informations exigées et évaluer l'information trouvée de manière critique.

La compétence d'identifier le besoin d'information comprend ces souscompétences :

- L'individu (apprenant/enseignant) doit savoir/reconnaître et caractériser son besoin d'information.

- Il doit comprendre l'objectif de sa recherche, et la portée et la pertinence des sources variées qu'il consulte lors de sa recherche d'informations.

- Il doit utiliser différentes sources pour vérifier les informations trouvées.

La compétence de produire et communiquer comporte ces souscompétences :

- Il doit savoir ordonner, classifier et stocker l'information trouvée.

- Il doit comprendre les différents enjeux éthiques, économiques, juridiques et sociaux qui sont relatifs à l'utilisation de l'information collectée.

- Il doit savoir comment mobiliser ses connaissances précédentes et nouvelles pour créer de nouvelles connaissances dans le domaine.

- Il doit savoir comment communiquer les résultats de sa réflexion de manière efficace.

La compétence d'accéder aux informations exigées d'une manière efficace englobe ces sous-compétences : 
- Il doit connaître les différents services d'assistance qui sont disponibles.

- Il doit savoir comment choisir les méthodes et les outils les plus adaptés pour obtenir l'information nécessaire.

- Il doit mobiliser les stratégies efficaces lors de la recherche de l'information.

La compétence d'évaluer l'information trouvée de manière critique conçoit ces sous-compétences :

- Il doit être capable d'évaluer la pertinence et l'importance de l'information trouvée.

- Il doit savoir comment définir et appliquer les critères pour évaluer l'information trouvée.

- Il doit être capable de réfléchir sur sa stratégie de recherche et de la modifier si elle n'est pas pertinente.

Quant à cette dernière compétence, le processus de l'évaluation et ses critères varient selon la forme du document, soit en papier ou numérique (Passerieux et Verreault (2013). Chaque jour, l'internet ne cesse pas de présenter des informations variées, similaires ou différentes, et qui abordent tous les secteurs de la vie. Certaines informations peuvent être correctes, pertinentes et fiables, alors que ce n'est pas le cas pour d'autres. L'application du Web 2.0 a parfaitement changé les critères de l'évaluation pour l'information trouvée sur ses sites Web (Sahut, 2017). Selon Oberhelman (2007), certains outils du cette version du Web comme les wikis, rendent les informations présentées évolutives et faciles à modifier par quiconque sans avoir considéré son expertise.

L'évaluation de l'information sur le Web est une démarche ayant pour but de vérifier, comparer et critiquer cette information de façon à extraire celle qui convient au contexte réel et aux attentes initiales du chercheur (Soung, 2017). Cette procédure est importante pour apprécier la crédibilité, l'accessibilité, la fraîcheur ou l'actualité de l'information par rapport à la recherche effectuée (Baschwitz et Antonia, 2010) et pour vérifier si cette information est valable, fiable et pertinente en ce qui concerne les besoins de la recherche (Passerieux et Verreault, 2013). Selon ces auteurs (Baschwitz et Antonia, 2010; Passerieux et Verreault, 2013; Soung, 2017), les critères considérés lors de cette évaluation sont la pertinence, la fiabilité, la réputation de l'auteur/le scripteur et la 
qualité du contenu présenté. À côté de ces critères, Mittermeyer et Quirion (2003) ajoutent la date de la publication de l'information et son exactitude.

On peut juger la pertinence de l'information en se basant sur quelques indicateurs en fonction du niveau d'information, de l'adéquation, du support graphique et de la méthodologie présentée (Archambault, Diane, Daphné et Jean-Yves, 2009). Selon Soung (2017) et sur le plan du niveau d'information, on doit examiner sa précision, sa clarté et le degré de l'argumentation indiquée; et quant à l'adéquation, le chercheur de l'information est la personne qui doit voir si l'information correspond à son besoin. D'après Pochet (2012), le support graphique indique le type de présentation de l'information qui consolide sa manifestation, soit un texte, un dessin, un tableau, etc. Pour le dernier indicateur, la méthodologie présentée, et comme le montre Gauthier (2009), elle peut se manifester dans les techniques utilisées pour atteindre l'objectif de recherche. Quant à la fiabilité de l'information, elle indique également à la crédibilité. Dans ce sens, et comme le montre Archambault, et al., (2009), afin d'examiner la crédibilité, le chercheur doit juger la présence physique de la source de l'information et l'apparence du site Web, et ce, par rapport à son intérêt lors de la recherche.

Concernant la réputation de l'auteur, elle est un des indicateurs les plus pertinents pour juger l'information en ligne. On peut examiner cet indicateur en s'appuyant sur l'affiliation, l'expertise, le numéro de citation et les différents renseignements biographiques présentés par l'auteur/le scripteur de l'information sur le site Web (Archambault, et al., 2009). L'évaluation de la qualité du contenu se base, enfin, sur l'examen de l'objectivité, de l'exactitude et de l'actualité de l'information trouvée sur le site Web (Soung, 2017).

\subsection{Les modèles de la formation informationnelle sur le Web}

Nombreux sont les modèles proposés par les chercheurs dans le domaine de la formation informationnelle des apprenants ou des futurs enseignants. Les lignes qui suivent présentent quatre modèles dans ce domaine : celui du Big6 Skills, celui de Kuhlthau, celui de 1'Association of College and Research Libraries (ACRL) et celui de Dumouchel (2016). 


\subsubsection{Le modèle du Big6 Skills ${ }^{1}$}

Le modèle du Big6 Skills de Mike Eisenberg et Bob Berkowitz est le modèle le plus répandu et le plus usé dans le domaine de l'enseignement de l'information et des compétences de la technologie. Il a été appliqué dans les curricula du primaire, du secondaire dans les programmes du développement des compétences des adultes. Le modèle se compose de six compétences essentielles qui sont les suivantes : 1) la définition de la tâche (définir le problème, identifier l'information demandée par le problème); 2) les stratégies de la recherche d'information (identifier de toutes les sources, choisir les sources pertinentes), 3) la localisation et l'accès (localiser les sources, trouver l'information dans les sources); 4) l'utilisation de l'information (lire et visionner l'information pour extraire la partie de l'information demandée); 5) la synthèse (organiser l'information trouvée, présenter l'information) et 6) évaluation (juger la production finale, juger le processus).

\subsubsection{Le modèle de Kuhlthau}

Le modèle du processus de recherche d'information de Kuhlthau (1993) est un autre modèle commun dans ce domaine de recherche. Il comprend six compétences comme telles: 1) l'initiation (avoir besoin d'une information); 2) la sélection (le choix d'un sujet à chercher); 3) l'exploration (trouver les informations profitables dans le cadre du sujet ciblé); 4) la formulation (construire une perspective personnelle à partir des informations trouvées); 5) la collection (recueillir les informations pertinentes) et 6) la présentation de l'information aperçue pour répondre à l'objectif de la recherche (Texte adapté de Kuhlthau, 2004).

\subsubsection{Le modèle d'"Association of College and Research Libraries (ACRL)"}

Le modèle de la Norme sur les compétences informationnelles dans l'enseignement supérieur de l'ACRL a été traduit en français en 2000 par la Conférence des recteurs et des principaux des universités du Québec

${ }^{1}$ Texte adapté de http://nmasse.com/courses/ref/big6/big6.htm qui est consulté le 17 novembre 2018. 
(2005). Ce modèle comporte cinq compétences à maîtriser, qui sont : 1) déterminer l'information dont on a besoin; 2) accéder à l'information ciblée de façon efficace; 3 ) évaluer de façon critique l'information et ses sources et intégrer cette information dans le réseau de connaissances; 4) utiliser l'information afin d'atteindre un objectif précis et 5) comprendre les différentes questions économiques, sociales et juridiques qui entourent l'utilisation de l'information, accéder à l'information et l'utiliser de manière éthique qui conforme à la loi (Texte adapté de CREPUQ, 2005).

\subsubsection{Le modèle de Dumouchel}

En analysant les modèles précédents et d'autres, Dumouchel (2016) montre que ces modèles comportent globalement cinq étapes: 1) identifier le besoin d'information, 2) élaborer des stratégies de recherche, 3) évaluer l'information, 4) utiliser l'information et 5) l'évaluation du processus de recherche d'information. Ces étapes sont les compétences principales les plus importantes qui peuvent permettre aux apprenants d'accéder à l'univers de l'information numérique. Selon le chercheur, ces modèles sont linéaires et ils ne considèrent pas l'aspect collaboratif dans la recherche. À l'instar de ces modèles et dans son projet doctoral en 2016, Dumouchel a présenté un modèle synthèse qui comprend cinq étapes itératives et possiblement collaboratives : 1) établir un besoin d'information, 2) chercher l'information, 3) évaluer l'information (l'autorité de l'information, la crédibilité de l'information, la qualité de l'information, la pertinence de l'information et la validation sociale de l'information), 4) utiliser l'information et 5) évaluer le processus effectué.

\subsection{Recension des études sur les compétences informationnelles}

Dans le domaine des TIC, l'étude de Walha et Peters (2011) a pour objectif de dégager les représentations sociales propres aux enseignantschercheurs tunisiens $(\mathrm{N}=64)$, et ceci à partir des actions déclarées. Pour atteindre l'objectif de l'étude, les chercheuses ont utilisé un questionnaire comportant 18 questions qui sont des deux types, ouvert et fermé. Les questions concernent les compétences pédagogiques et techniques et l'utilisation des TIC chez les enseignants. L'analyse qualitative et quantitative des réponses présentées par les enseignants participants a montré que leur intérêt est porté sur le processus de 
l'enseignement, et que leurs représentations quant à l'usage des TIC sont complexes et variées et qui unissent des éléments de modernisation de l'acte d'enseigner et des freins aux pratiques.

Giroux, Gagnon, Gremion et Heinzen (2014) ont mené une étude collaborative qui vise à vérifier les croyances de futurs enseignants en ce qui concerne la pertinence des compétences des TIC. L'étude vise également à examiner leur perception du niveau de la responsabilité quant au développement de ces compétences chez les élèves. Afin d'atteindre ces deux objectifs, les chercheurs ont élaboré un questionnaire destiné aux étudiants de l'université $(\mathrm{N}=823)$ dans trois différentes universités au Québec, en Suisse et en France. Le questionnaire a compris 21 compétences proposées en TIC comme : l'utilisation efficace du courriel, l'utilisation efficace d'un logiciel de traitement du texte, l'utilisation efficace d'un tableur, l'utilisation efficace d'un logiciel de présentation, la modification des images et des phonographes numériques, la validation de l'information trouvée, l'édition d'un blogue et l'édition d'un wiki, etc. Le questionnaire a examiné, en même temps, les opinions de futurs enseignants concernant des responsables d'encadrement et du développement de ces compétences considérées, soit les parents, les écoles, les élèves euxmêmes ou les entreprises. Les résultats de l'étude indiquent la présence de quelques différences qui caractérisent les étudiants participants quant à l'importance des compétences des TIC, ce qui peut être dû à la différence de leurs pays et de leurs intérêts éducatifs, politiques, économiques, etc. En outre, leurs perceptions du niveau de la responsabilité du développement des compétences chez les élèves sont également variées. Néanmoins, les enseignants voient qu'une bonne part de cette responsabilité doit revenir aux familles et aux élèves euxmêmes.

Pour sa part, Dumouchel (2016) a visé à décrire les pratiques des futurs enseignants québécois en recherche d'informations, à analyser ces pratiques en traitement de l'information et à comprendre comment les futurs enseignants sont formés pour enseigner les compétences informationnelles à leurs élèves lors de la pratique de leur métier. Afin d'atteindre ces objectifs, le chercheur a élaboré un questionnaire ayant des questions des deux types fermé et ouvert; il a effectué des entretiens 
individuels avec 9 futurs enseignants et il a appliqué des exercices de recherche et de traitement d'information avec les enseignants rencontrés. L'analyse des données recueillies lors de ces différents outils de recherche montre que la plupart des futurs enseignants fait de novices, planifie peu ou pas leur processus de recherche, utilise des stratégies basiques lors de la recherche en employant le Google dans la plupart des cas de la recherche. L'analyse montre également que la majorité de ces enseignants réalise une évaluation pour les informations trouvées sur le Web et que la recherche d'informations se réalise à des fins de leurs études universitaires. L'étude indique, en fin, que la formation de ces enseignants dans le cadre de leur université n'est pas suffisante, ce qui ne leur permettra pas de bien enseigner les compétences informationnelles à leurs élèves au futur.

Dans le domaine de l'évaluation des informations numériques, l'étude de Soung (2017) a pour but de décrire les différentes représentations des étudiants universités québécois quant aux critères d'évaluation de l'information en ligne. Pour ce faire, la chercheuse a posé un questionnaire aux 268 étudiants et elle a réalisé des rencontres semidirigées avec 54 étudiants. L'analyse des données obtenues montre des taux de réponse qui sont intéressants dans les quatre éléments principaux considérés, la pertinence de l'information, la fiabilité des ressources utilisées, la réputation de l'auteur/l'écrivain et enfin, la qualité du contenu trouvé.

À la lumière de ces études déjà mentionnées, on peut conclure que les questionnaires et les rencontres individuelles sont les outils de la recherche les plus utiles et les plus pertinents pour aborder les compétences informationnelles chez les enseignants. En s'inspirant de ces études, la partie suivante présente les choix méthodologiques considérés et mobilisés dans le cadre de l'étude actuelle : l'échantillon, les instruments et l'approche utilisée lors de l'analyse des données.

\section{Méthodologie}

De façon générale, trois outils différents sont élaborés et utilisés dans cette étude descriptive: une grille proposée pour identifier les compétences informationnelles nécessaires à la formation contenue des enseignants dans le contexte égyptien, un questionnaire et un entretien 
individuel et semi-dirigé avec un certain nombre des enseignants en poste du FLÉ pour savoir le degré de leur maîtrise pour les compétences traitées. Pour vérifier la validité des outils suggérés, ils ont été évalués par un jury composé de 12 membres : 5 professeurs à la faculté de l'éducation, 6 enseignants du français et une bibliothécaire ${ }^{2}$. Après avoir évalué les outils, une étude pilote a été menée avec cinq professeurs pour confirmer la pertinence et la clarification des items des outils. L'annexe 1 présente les membres du jury en détail. Lors de l'examen des données recueillies, une analyse quantitative ainsi que qualitative a été relaissée.

\subsection{L'échantillon de l'étude}

L'échantillon de l'étude comporte 45 enseignants (es) actuels du FLÉ. Ils sont variés en ce qui concerne le nombre des années d'expertise : 28 enseignants (es) novices (moins de 5 ans d'expérience en enseignement) et 17 enseignants (es) experts (plus de 5 ans d'expérience en enseignement). En outre, ils sont des enseignants dans deux différents cycles éducatifs, préparatoire et secondaire. L'annexe 2 montre les données relatives à ces enseignants.

\subsection{Les instruments de l'étude}

Afin de répondre à la première question de l'étude, une grille a été élaborée pour déterminer les compétences informationnelles que les enseignants doivent maîtriser dans la société du savoir. La grille a été construite après de lectures variées dans la littérature et dans les travaux menés sur ce domaine de recherche, en particulier l'étude de Dumouchel (2016). La première version de la grille a compris cinq domaines principaux des compétences ayant 52 sous-compétences. Il existe, devant chaque sous-compétence, trois choix : 1) convenable, 2) non convenable, 3) commentaire proposé. Selon l'évaluation menée, six souscompétences n'étaient pas convenables, huit sous-compétences étaient générales et trois autres étaient ambiguës. Après avoir enlevé les souscompétences non convenables et modifié les sous-compétences générales et ambiguës selon les opinions des membres du jury, la grille a composé 48 sous-compétences dans le cadre de cinq compétences principales.

Pour répondre à la deuxième question de l'étude qui exige de déterminer le degré de la maîtrise des compétences informationnelles auprès des enseignants actuels du FLÉ, deux instruments ont été appliqués. Le 
premier est un questionnaire destiné à un échantillon des 45 enseignants du FLÉ et dont les données se présentent dans l'annexe 2. Dans cette perspective, et comme le montre (Rasmy, 2010), le questionnaire est l'instrument de mesure qui est le plus mobilisé dans la littérature afin d'étudier la présence des compétences informationnelles auprès des personnes participantes à l'étude. Ce questionnaire a été élaboré selon les compétences informationnelles présentées dans la grille proposée. Il a été évalué par le même jury de 12 membres précédents.

Après la modification et dans sa dernière version, le questionnaire a compris deux domaines primordiaux : les compétences informationnelles en général et les compétences informationnelles sur le Web. Le premier a comporté 12 sous-compétences informationnelles en général. Le second s'est divisé en quatre compétences principales: la reconnaissance d'un besoin d'information sur le Web ayant 6 sous-compétences, la recherche de l'information sur le Web comportant 8 sous-compétences, l'évaluation de l'information trouvée sur le Web comportant 11 souscompétences et l'utilisation de l'information trouvée sur le Web avec 9 sous-compétences. Pour vérifier la cohérence interne du questionnaire, on a calculé la valeur de référence de l'Alpha de Cronbach. Celle-ci était 0.87 , ce qui montre une bonne cohérence interne pour ses différents items (Miles et Huberman, 2003).

Afin de répondre aux items du questionnaire, l'enseignant du FLÉ doit choisir une réponse auprès d'une échelle à 4 niveaux : pas compétent $\left(\mathrm{n}^{\mathrm{o}}\right.$ $1)$, débutant $\left(n^{\circ} 2\right)$, moyen $\left(n^{\circ} 3\right)$, compétent $\left(n^{\circ} 4\right)$. Les moyennes et les déviations standards ont été calculées pour chaque compétence principale et pour l'ensemble de compétences abordées dans le questionnaire. En se basant sur la valeur des scores moyens correspondant aux items du questionnaire, il existe quatre niveaux des valeurs à considérer (Robert et Bouillaguet, 1997) :

- La valeur de (1.00) à (1.75) : l'enseignant n'est pas compétent par rapporte à la compétence abordée.

- La valeur de (1.76) à (2.50) : le niveau de l'enseignant est débutant par rapporte à la compétence abordée.

- La valeur de (2.51) à (3.25) : le niveau de l'enseignant est moyen par rapporte à la compétence abordée. 
- La valeur de (3.26) à (4.00) : l'enseignant est compétent par rapporte à la compétence abordée.

Afin d'obtenir un portrait plus détaillé des réponses et des niveaux des enseignants participants en ce qui concerne la maîtrise des compétences abordées, des entretiens semi-dirigés ont été menés de manière individuelle avec 15 enseignants (es), ce qui constitue le second instrument. Sur le plan méthodologique, l'entretien semi-dirigé est une « interaction verbale animée de façon souple par le chercheur » (SavoieZjac, 2009, p.340). Il vise à faire une discussion entre le chercheur et la personne interviewée dans certains thèmes étudiés en donnant à cette personne l'opportunité de s'exprimer et expliciter librement ses propos (Gauthier, 2007). Ces entretiens ont été menés à partir des programmes de l'interaction sociale sur le Web (Watts App et Messenger). Chaque rencontre a pris de 15 à 20 minutes, et ceci, selon les réponses des personnes participantes. Afin de vérifier la validation de l'entretien proposé, le protocole a été l'objet d'une validation d'une étude pilote avec cinq enseignants.

Cette procédure a permis de réaliser certaines modifications en ce qui concerne la formulation et la clarté des questions posées, notamment dans la dernière question.

Après la modification, le protocole de l'entretien semi-dirigé a commencé par une explication de l'objectif de l'étude et de l'entretien mené et l'assurance de la confidentialité des réponses présentées. L'entretien s'est composé de cinq questions. La première vise à savoir des informations personnelles comme le nom de l'enseignant, l'école où il travaille, les années de l'expérience et le degré professionnels. Ceci a permis d'établir une bonne relation avec la personne et de lui encourager à répondre aux questions suivantes. La deuxième question vise à savoir quel outil l'enseignant utilise le plus souvent pour obtenir des informations dans son travail/métier. La troisième question ayant le but de savoir dans le cas de l'utilisation de l'internet pour chercher des informations, quelles sont les étapes à suivre pour mener cette recherche. La quatrième question concerne l'évaluation des informations trouvées en ligne. Celle-ci demande aux enseignants s'ils évaluent les informations trouvées lors de leur recherche de l'information, et dans le cas de la réponse "oui”, l'enseignant doit mentionner les procédures à 
suivre. La cinquième question vérifie la compréhension des enseignants en ce qui concerne la différence entre le plagiat et la citation, une des sous-compétences présentées dans le questionnaire. Dans l'étude pilote, certains enseignants n'ont pas compris cette différence, ce qui a mené la chercheuse à modifier la question pour être une question à choix multiple. Dans cette perspective, cinq réponses ont été proposées aux enseignants. La question vise alors à savoir comment faire dans le cas de vouloir prendre/écrire une information trouvée en ligne. L'enseignant peut choisir une seule réponse : 1) il écrit l'information telle que sans mentionner la source; 2) il écrit l'information en mentionnant la source; 3) il écrit l'information en faisant des changements sans mentionner la source; 4) il écrit l'information en faisant des changements et en mentionnant la source ou 5) une autre réponse (dans ce dernier cas, l'enseignant doit présenter sa propre réponse).

\section{Résultats de l'étude}

\subsection{Les compétences informationnelles}

Après avoir modifié la grille proposée, sa dernière version montre que les compétences informationnelles nécessaires à la formation contenue des enseignants égyptiens dans le monde du savoir comportent cinq domaines essentiels ayant 46 sous-compétences et qui sont: 1) les compétences informationnelles en général, 2) la reconnaissance d'un besoin d'information sur le Web, 3) la recherche de l'information sur le Web, 4) l'évaluation de l'information trouvée sur le Web et 5) l'utilisation de l'information trouvée sur le Web. Dans le $1^{\text {er }}$ domaine, les compétences informationnelles en général, la grille comporte 12 éléments pour obtenir les informations : 1) utiliser le manuel scolaire, 2) utiliser le livre du maître «le guide pédagogique », 3) utiliser des manuels extérieurs, 4) aller à la bibliothèque de l'université, de l'école, etc., 5) consulter le superviseur «le directeur», 6) consulter les enseignants les plus âgés, 7) utiliser l'internet à partir d'un ordinateur/portable/blocs-notes/tablette, etc., 8) utiliser les programmes de l'interaction sociale: le Facebook, le Watts App, le Twitter ou Instgrame, etc., 9) naviguer les sites Web éducatifs, 10) consultez des sites Web liés au ministère de l'éducation et de l'enseignement, 11) utilisez des supports électroniques (ex. : dictionnaire en ligne, livres PDF en grammaire ou en phonétique, etc.) et 12) lire des articles scientifiques 
dans le domaine de l'enseignement/apprentissage du français pour enrichir les informations actuelles.

Dans le $2^{\mathrm{e}}$ domaine, celui de la reconnaissance d'un besoin d'information sur le Web, la grille contient 6 sous-compétences, qui sont: 1) pouvoir préciser le besoin d'information, 2) comprendre l'objectif de la recherche d'information, 3) connaître des sources variées à consulter, 4) avoir une ou plus d'une méthode « façon/procédure » pour chercher l'information, 5) pouvoir formuler une question pour chercher l'information et 6) pouvoir identifier de bons mots clés, des synonymes ou des termes associés au besoin d'information pour mener la recherche demandée.

Quant au $3^{\text {e }}$ domaine, la recherche de l'information sur le Web, la grille comprend les 8 sous-compétences suivantes : 1) utiliser des sites Web variés d'information, notamment dans le domaine éducatif, 2) utiliser des sources variées (blogue, vidéo, capsule audio, etc.), 3) utiliser le Google, 4) utiliser le Google scholar, 5) utiliser le Wikipédia, 6) utiliser le YouTube, 7) participer à des groupes sur les sites de l'interaction sociale : Watts App, Twitter, Facebook, Instagram, etc., et 8) connaître les sites Web qui présentent des informations pertinentes et qui répondent efficacement au besoin informationnel.

Concernant l'évaluation de l'information trouvée sur le Web, le $4^{\mathrm{e}}$ domaine, 11 sous-compétences se trouvent dans la grille, telles que : 1) pouvoir choisir la réponse la plus pertinente correspondante à la question posée, 2) pouvoir choisir l'information la plus récente à partir de lire la date de la mise à jour du contenu trouvé en ligne, 3) vérifier l'auteur ou le scripteur de l'information trouvée, 4) pouvoir chercher l'information sur plusieurs sites Web et ne pas contenter de la première page apparue dans la recherche menée, 5) pouvoir distinguer entre les vérités et les opinions en lisant les informations en ligne, 6) pouvoir critiquer les opinions trouvées et les informations incorrectes, 7) pourvoir vérifier l'adresse électronique (http://www....) relative à l'information trouvée, 8) pouvoir changer la méthode «procédure » de recherche en fonction de la qualité ou de la pertinence de l'information demandée, 9) pouvoir étendre la recherche des informations si l'information trouvée est incomplète, 10) pouvoir reformuler la question de la recherche si l'information obtenue n'est pas appropriée au besoin informationnel et 
11) connaître les différents critères pour évaluer l'information trouvée en ligne.

Enfin, le $5^{\mathrm{e}}$ domaine, celui de l'utilisation de l'information trouvée sur le Web, comporte 9 sous-compétences comme suit: 1) connaître la différence entre le plagiat et la citation, 2) connaître les méthodes d'éviter le plagiat, 3) comprendre les enjeux de la propriété culturelle des auteurs/scripteurs, 4) mentionner la référence/la source de l'information : le nom de l'auteur, la date de la publication, le site Web, etc., 5) lire et exploiter les références bibliographiques citées par l'auteur s'il y a lieu, 6) connaître les règles d'utiliser et d'intégrer l'information dans un document, 7) partager l'information avec les collègues ou avec les personnes intéressées, 8) réutiliser l'information dans de nouveaux contextes et 9) pouvoir mobiliser les informations et les connaissances antérieures et trouvées pour produire d'autres nouvelles.

\subsection{La maîtrise des compétences informationnelles auprès des enseignants du FLÉ}

Afin de répondre à la $2^{\mathrm{e}}$ question de l'étude qui exige de savoir le degré de la maîtrise des compétences informationnelles considérées chez les enseignants actuels du FLÉ, le tableau 1 présente les moyennes et les déviations standards des scores présentés par les enseignants lors de la réponse au questionnaire posé.

\section{Tableau 1: Les compétences informationnelles chez les enseignants actuels du FLÉ} Compétences à considérer

Moyenne \pm déviation standard

1. Les compétences informationnelles en général

$2.52 \pm 1.06$

2. Les compétences de la reconnaissance d'un besoin $3.42 \pm 0.71$ d'information sur le Web

3. Les compétences de la recherche de l'information sur le Web

4. Les compétences de l'évaluation de l'information $2.49 \pm 0.89$ sur le Web

5. Les compétences de l'utilisation de l'information sur le Web 
Comme le montre ce tableau, le niveau des enseignants participants est moyen dans la maîtrise de l'ensemble des compétences informationnelles considérées. Puisque, selon les valeurs considérées dans l'étude, le moyen des scores présentés est de 2.65 et qui se trouve entre 2.51 et 3.25. Les enseignants sont compétents dans la reconnaissance du leur besoin d'information sur le Web; ils ont un niveau moyen dans la recherche de l'information sur le Web et dans les compétences informationnelles générales; alors que leur niveau est encore débutant dans l'évaluation et l'utilisation de l'information sur le Web. Ces résultats sont également introduits dans la figure 1.

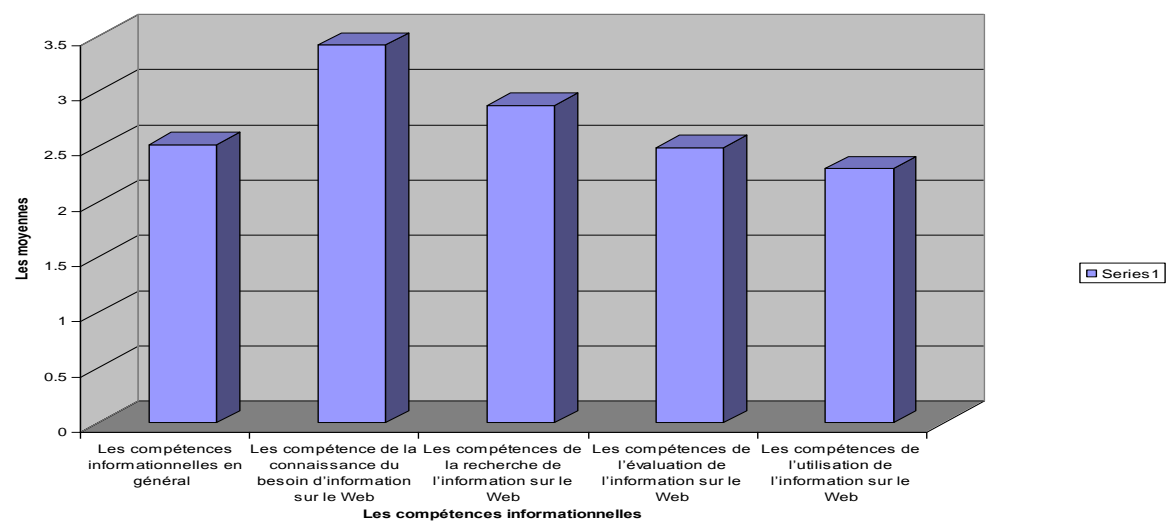

Figure 1 : la maîtrise des compétences informationnelles auprès des enseignants du FLÉ

\section{Tableau 2 : Les compétences informationnelles en général}

Sources d'information à considérer

Moyenne \pm déviation standard

1. Vous utilisez le manuel scolaire.

$2.70 \pm 1.21$

2. Vous utilisez le livre du maître (le guide

$2.25 \pm 1.20$ pédagogique).

3. Vous utilisez des manuels extérieurs.

4. Vous allez à une bibliothèque : à l'école, à 
l'université, à la bibliothèque de votre gouvernorat, etc.

5. Vous consultez votre superviseur (le directeur ou $\quad 2.10 \pm 1.16$ le premier maître à l'école).

6. Vous consultez les enseignants les plus âgés. $\quad 2.50 \pm 1.19$

7. Vous utilisez l'internet à partir d'un $3.30 \pm 0.86$ ordinateur/portable/blocs-notes/tablette, etc.

8. Vous utilisez un compte personnel sur le $\quad 2.65 \pm 1.22$

Facebook, le Watts App, le Twitter ou l'Instagrame, etc.

9. Vous accédez aux sites Web éducatifs en général $\quad 2.90 \pm 1.11$ sur l'internet.

10. Vous consultez des sites Web liés au ministère de $\quad 2.00 \pm 0.91$ l'éducation et de l'enseignement.

11. Vous utilisez des supports électroniques (ex. : $\quad 2.65 \pm 1.03$ dictionnaire en ligne, livres PDF en grammaire, en phonétique, etc.)

12. Vous lisez des articles scientifiques dans le $\quad 1.75 \pm 0.44$ domaine de l'enseignement/apprentissage du français pour enrichir vos informations. L'ensemble de sources d'information

Quant aux compétences informationnelles qui sont en général, le tableau (2) montre que les enseignants utilisent souvent l'internet à partir de leurs ordinateurs/portables/blocs-notes/tablettes, etc. De façon moyenne, et pour obtenir des informations dans leur métier, ils ont recours aux sites Web éducatifs, aux manuels extérieurs, au manuel scolaire, aux supports électroniques, aux enseignants les plus âgés, au livre du maître, aux superviseurs/directeurs, aux enseignants les plus âgés et aux sites Web du ministère de l'éducation et de l'enseignement. Ils se réfèrent du temps en temps aux bibliothèques et à la lecture des articles scientifiques dans le domaine de leur travail. 


\section{Tableau 3 : Les compétences de la reconnaissance d'un besoin d'information sur le Web}

\section{Sous-compétences à considérer}

1. Vous pouvez préciser votre besoin d'information.

2. Vous comprenez l'objectif de votre recherche d'information.

3. Vous connaissez des sources variées à consulter.

$3.30 \pm 0.80$

4. Vous avez une ou plus d'une méthode/façon/procédure pour chercher l'information demandée.

5. Vous pouvez formuler une question pour chercher l'information.

6. Vous pouvez identifier de bons mots clés, des$$
3.15 \pm 0.74
$$$$
3.50 \pm 0.60
$$

$3.75+0.44$ déviation synonymes ou des termes associés à votre besoin d'information.

L'ensemble de sous-compétences

Comme le montre le tableau (3) relatif à la reconnaissance d'un besoin d'information sur le Web, les enseignants sont compétents dans la plupart des sous-compétences. Ceci apparaît clairement dans l'identification de mots clés associés à leur besoin d'information, la précision d'un besoin d'information, la compréhension de l'objectif de la recherche d'informations et la connaissance des sources variées à consulter. En outre, leur niveau moyen est assez près de la maîtrise en ce qui concerne le fait d'avoir de différentes manières pour chercher l'information et la formulation de bonnes questions lors de la recherche ciblée.

Le tableau 4 : Les compétences de la recherche de l'information sur le Web

Sous-compétences à considérer 
1. Vous utilisez des sites Web variés d'informations, $3.35 \pm 0.67$ notamment dans le domaine éducatif.

2. Vous utilisez des sources variées (blogue, vidéo, $3.35 \pm 0.48$ capsule audio, etc.)

3. Vous utilisez le Google.

$3.75 \pm 0.71$

4. Vous utilisez le Google scholar.

$2.00 \pm 1.16$

5. Vous utilisez le Wikipédia.

$2.00 \pm 1.25$

6. Vous utilisez le YouTube.

$3.50 \pm 0.60$

7. Vous participez à des groupes sur les sites de

$2.65 \pm 1.26$

l'interaction sociale : le Watts App, le Twitter, le Facebook, 1'Instagram, etc.

8. Vous connaissez les sites Web qui présentent des informations pertinentes et qui répondent à votre besoin informationnel.

L'ensemble de sous-compétences

$2.87 \pm 1.15$

Quant aux compétences de la recherche de l'information en ligne, le tableau 4 indique que les enseignants sont compétents en ce qui traite à utiliser le Google, le YouTube, les sites Web variés notamment en éducation et les autres sources (blogue, vidéo, capsule audio, etc.). Ils ont un niveau moyen dans la participation aux groupes de l'interaction sociale : le Watts App, le Twitter, le Facebook, l'Instagram, etc. Pour l'utilisation des sites Web présentant des informations pertinentes, comme le Google scholar et le Wikipédia, le niveau des enseignants est encore débutant.

\section{Tableau 5 : Les compétences de l'évaluation de l'information sur le Web}

\section{Sous-compétences à considérer \\ Moyenne \pm déviation standard}

1. Vous choisissez la réponse la plus pertinente à $3.95 \pm 0.22$ votre question.

2. Vous choisissez l'information la plus récente à $2.65 \pm 1.26$ partir de lire la date de la mise à jour du contenu trouvé en ligne.

3. Vous vérifiez l'auteur/le scripteur de $\quad 1.90 \pm 0.71$ l'information trouvée.

4. Vous cherchez l'information sur plusieurs sites $\quad 2.45 \pm 0.82$ 
Web et vous ne contentez pas de la première page apparue dans votre recherche.

5. Vous distinguez entre les vérités et les opinions $\quad 2.80 \pm 1.00$ en lisant les informations en ligne.

6. Vous critiquez les opinions trouvées et les $\quad 2.80 \pm 1.05$ informations incorrectes.

7. Vous vérifiez l'adresse électronique $\quad 2.45 \pm 0.94$ (http://www...) pour l'information.

8. Vous changez la méthode de recherche en $\quad 2.50 \pm 0.94$ fonction de la qualité ou de la pertinence de l'information demandée.

9. Vous étendez la recherche des informations si $\quad 2.65 \pm 0.81$ l'information trouvée est incomplète.

10. Vous reformulez la question de la recherche si $\quad 2.55 \pm 0.68$ l'information obtenue n'est pas appropriée à votre besoin.

11. Vous connaissez les différents critères pour $\quad 2.00 \pm 0.72$ évaluer l'information trouvée.

L'ensemble de sous-compétences

Le tableau (5), en présentant les compétences de l'évaluation de l'information sur le Web, indique que les enseignants sont compétents à choisir des réponses pertinentes à leurs questions posées. Il indique également que leur niveau est moyen dans de nombreuses souscompétences: distinguer entre les vérités et les opinions, critiquer les opinions et les informations présentées, choisir les informations les plus récentes, étendre et reformuler les questions de la recherche si les informations trouvées sont incomplètes. Néanmoins, en ce qui concerne ces sous-compétences : changer la manière de recherche en fonction de la qualité/la pertinence de l'information, vérifier l'adresse électronique des informations obtenues, connaître les critères de l'évaluation de l'information et vérifier l'auteur/le scripteur de cette information, le niveau des enseignants est encore débutant. 
Les compétences informationnelles nécessaires à la formation contenue................

\section{Tableau 6: Les compétences de l'utilisation de l'information sur le Web}

Sous-compétences à considérer

Moyenne \pm déviation standard

1. Vous connaissez la différence entre le plagiat et $2.00 \pm 0.91$ la citation.

2. Vous connaissez les méthodes d'éviter la production du plagiat.

3. Vous comprenez les enjeux de la propriété

$2.15 \pm 0.98$ culturelle des auteurs.

4. Vous mentionnez la référence de l'information : $\quad 1.95 \pm 1.05$ le nom de l'auteur, la date de la publication, le site Web, etc.

5. Vous lisez et exploitez des références bibliographiques citées par l'auteur, s'il y a lieu.

6. Vous connaissez les règles d'utiliser et d'inclure $2.45 \pm 0.60$ l'information dans votre document.

7. Vous partagez l'information avec vos collègues. $\quad 3.05 \pm 0.82$

8. Vous réemployez l'information dans de $2.80 \pm 0.89$ nouveaux contextes.

9. Vous pouvez mobiliser les informations trouvées pour produire d'autres nouvelles.

L'ensemble de sous-compétences

Selon les résultats des compétences de l'utilisation de l'information en ligne et qui sont présentés dans le tableau 6, les enseignants se distinguent par un niveau moyen dans trois sous-compétences : réutiliser l'information dans d'autres contextes, mobiliser les informations trouvées afin de produire d'autres nouvelles et partager ces informations avec les collègues. Ils ont un niveau débutant dans le reste de souscompétences abordées : connaître les règles d'inclure l'information dans un document, comprendre les enjeux de la propriété culturelle des auteurs, connaître les méthodes d'éviter le plagiat, connaître la différence entre le plagiat et la citation, citer les références relatives à l'information trouvée et exploiter des références bibliographiques présentées par l'auteur. 


\section{Les entretiens individuels et semi-dirigés avec les enseignants}

L'analyse des données obtenues lors de la réalisation des entretiens individuels avec les enseignants a enrichi les résultats précédents en ajoutant un certain nombre de remarques pertinentes. Lors du besoin de la recherche de l'information, plus de la moitié des enseignants participants (60\%) préfèrent l'utilisation de l'internet (9/15 enseignants). Puisqu'il leur semble que l'internet est l'outil le plus proche, le plus facile et le plus rapide à consulter. De plus, selon eux, l'internet leur présente plusieurs réponses, plusieurs exemples et des explications détaillées pour leurs questions posées, ce qui les amène à préférer la navigation sur ses sites Web. Quatre enseignants (4/15 enseignants; $26.67 \%$ ) préfèrent la question d'autres personnes, notamment les collègues les plus âgés. Selon eux, ils rencontrent leurs collègues tout le temps à l'école et ils parlent avec eux de leurs questions en obtenant les réponses demandées dans la même rencontre, et cette façon est la plus facile pour eux. Alors que deux enseignants préfèrent de fréquenter les bibliothèques pour la recherche des informations (2/15 enseignants;13.33\%). Ces deux enseignants ont recours aux bibliothèques pour obtenir des informations authentiques, et ce, selon leur point de vue.

Dans le cas de l'usage de l'internet pour trouver des informations, $53.33 \% \quad(8 / 15$ enseignants) utilisent le Google; 26.67\% (4/15 enseignants) utilisent le YouTube et 20\% (3/15 enseignants) utilisent ces deux moteurs de la recherche. Selon ces enseignants, le Google et les YouTube sont les deux moteurs les plus proches, les plus connus et les plus variés dans les résultats présentés. Dans cette perspective, l'ensemble des enseignants confirme le fait de suivre presque les mêmes étapes qui sont : écrire les mots-clés ou formuler une question sur l'écran de leurs appareils électroniques; lire le premier résultat de la recherche; dans le cas de ne pas trouver la réponse demandée où l'information trouvée est incomplète, ils examinent le deuxième résultat, puis le troisième jusqu'à l'obtention de l'information demandée. Ils ont confirmé que ce sont les étapes connues chez toutes les personnes.

Dans le cas de vouloir utiliser l'information trouvée en ligne dans leur travail, $46.67 \%$ des enseignants (7/15 enseignants) ne s'intéressent pas à 
l'évaluation de cette information; $53 \%$ des enseignants (8/15) s'occupent de l'évaluation, et dans ce cas ils se divisent en deux groupes : le premier constitue $26 \%$ (4/15 enseignants) et qui consulte les collègues pour vérifier la pertinence de l'information, alors que le second (26\%:4/15 enseignants) cherche l'information dans d'autres sites Web pour confirmer l'information. Ils évaluent ainsi l'information trouvée en ligne par deux moyens : la question d'autres collègues, notamment les plus experts, et la recherche dans d'autres sites Web. Ils n'ont pas d'autres critères à utiliser pour évaluer ces informations. Alors que les autres ne considèrent pas le processus de l'évaluation, puisqu'ils voient que les informations trouvées sur le Web sont correctes et qu'ils n'ont pas besoin de confirmer leur pertinence. Ils considèrent l'internet une source pertinente pour les besoins informationnels.

Afin de citer et écrire l'information trouvée en ligne, $80 \%$ des enseignants (12/15 enseignants) écrivent l'information sans mentionner la référence/la source et cette catégorie comporte deux groupes : le premier constituant $53.33 \%$ (8/15 enseignants) écrit la même information sans mentionner la référence et le second comportant $26.67 \%$ (4/15 enseignants) reformule l'information sans mentionner la référence. Seuls trois enseignants (20\%) utilisent l'information en ajoutant la référence/la source. Pour ceux qui écrivent l'information sans mentionner la référence/la source, ils voient que les informations trouvées sur le Web sont à la portée de tous et ils se demandent pourquoi on mentionne des sources connues et trouvées tout le temps sur les écrans de l'internet. Alors que les autres enseignants qui écrivent les références utilisées voient qu'il faut respecter la propriété culturelle des auteurs et ils comprennent les conceptions du plagiat et de la citation lors de l'écriture sur le Web.

\section{Discussion}

La présente étude avait pour but d'identifier les compétences informationnelles que les enseignants doivent avoir et de vérifier si les enseignants du FLÉ possèdent ces compétences d'une manière suffisante pour qu'ils puissent pratiquer leur métier et pour qu'ils puissent en enseigner à leurs élèves. Afin d'atteindre les objectifs de l'étude, une grille des compétences, un questionnaire et des entretiens semi-structurés ont été élaborés et appliqués. Les résultats de l'étude identifient cinq 
domaines primordiaux des compétences à maîtriser : des compétences générales qui sont relatives à l'information trouvée en papier et sous une forme numérique, des compétences de la reconnaissance d'un besoin informationnel, des compétences de la recherche de l'information, des compétences de l'évaluation de l'information et celles de l'utilisation de l'information, et ce, sur le Web. Les résultats relatifs aux quatre compétences informationnelles sur le Web s'accordent parfaitement avec la définition de ces compétences présentée par la CREPUQ (2005) et avec les modèles de la formation informationnelle, le Big6 Skills, celui de Kuhlthau, celui de 1'Association of College and Research Libraries (ACRL) et celui de Dumouchel (2016).

Quant à la présence des compétences informationnelles chez les enseignants participants, l'analyse des données obtenues du questionnaire indique qu'ils maîtrisent l'ensemble de compétences abordées d'un degré moyen. Ils sont compétents dans la reconnaissance $\mathrm{du}$ leur besoin d'information sur le Web; ils sont moyens dans les compétences informationnelles générales et dans la recherche de l'information sur le Web, alors que leur niveau en ce qui trait à l'évaluation et à l'utilisation de l'information sur le Web est encore débutant. Dans les compétences générales de l'information, ils préfèrent l'utilisation de l'internet, des sites Web éducatifs plus que les autres outils, ce qui indique l'importance de l'internet chez eux comme le premier outil à consulter dans leur métier, et ceci indique également le transfert de l'information en papier vers l'information numérique dans les intérêts de ces enseignants.

En outre, il semble que les enseignants connaissent bien déterminer leurs besoins informationnels, préciser les objectifs de la recherche, proposer des mots clés, formuler des questions, avoir des sources et des méthodes variées pour commencer la recherche des informations demandées. Lors de cette recherche, ils préfèrent l'utilisation du Google et le YouTube qui sont les deux moteurs de recherche les plus connus et les plus répandus. Bien qu'ils connaissent bien d'autres outils comme le Google scholar, le Wikipédia, etc., leur niveau dans l'ensemble des compétences de la recherche de l'information est moyen. On peut interpréter ce niveau par l'utilisation permanente du Google et de YouTube afin de répondre à leurs besoins informationnels. Ce résultat concorde avec celui de 
Dumouchel (2016). Ce dernier a montré que le Google est le moteur le plus mobilisé par les étudiants universitaires dans la recherche d'informations.

En ce qui concerne l'évaluation de l'information numérique, il semble que ces enseignants sont compétents à choisir l'information la plus pertinente et qui satisfait leur besoin. Ils ont un niveau moyen à distinguer entre les vérités et les opinions, à critiquer les opinions présentées, à choisir l'information la plus récente, à reformuler la question de la recherche, à étendre la recherche et à changer la méthode de recherche s'il est nécessaire. Alors qu'ils ne s'intéressent pas à vérifier l'adresse électronique ou l'auteur/le scripteur de l'information et ils ne connaissent pas comment évaluer l'information et quels critères à utiliser s'ils veulent mener cette évaluation. Ce résultat ne concorde pas avec l'étude de Dumouchel (2016), dans laquelle la plupart des sujets réalise une évaluation pour les informations trouvées sur le Web, et ce, dans le cadre de son étude.

Quant aux compétences de l'utilisation de l'information numérique, le niveau des enseignants participants est encore débutant. Puisqu'ils ont une difficulté à comprendre la différence entre le plagiat et la citation, les méthodes d'éviter le plagiat, les droits de la propriété culturelle des auteurs et ils ne s'intéressent pas à citer la référence ou la source de l'information obtenue. Il semble clairement qu'ils ont un manque dans les compétences relatives à ce domaine. Ces conséquences ne sont pas en accord avec celles de Dumouchel (2016). Par rapport à l'évaluation et à l'utilisation de l'information sur le Web, la différence entre les résultats de l'étude actuelle et ceux de Dumouchel (2016) revient peut-être aux universités canadiennes. Ces dernières appliquent toujours des règles précises dans le processus de l'évaluation des travaux pratiques et théoriques demandés de la part des étudiants et des chercheurs, ce qui les fait plus sensibles à évaluer leurs informations trouvées et à faire attention à commettre le plagiat dans leurs écrits.

Les résultats du questionnaire ont été confirmés par ceux des entretiens semi-dirigés, puisque plus de la moitié des enseignants participants préfèrent l'internet en utilisant le Google et le YouTube lors de la recherche d'informations. En outre, presque la moitié des enseignants 
interviewés ignorent l'évaluation de l'information obtenue sur le Web et la plupart des enseignants écrit l'information trouvée sans mentionner la référence (l'auteur/le scripteur, le titre du document, l'adresse électronique, etc.). Ces enseignants voient que l'internet est un outil efficace, rapide et facile à mobiliser et que les informations trouvées sur les sites Web sont variées et à la portée de tous, ce qui les amène à ne pas citer les références interrogées. Seuls trois enseignants voient la nécessite de citer les références consultées. Ceci revient au fait que ces enseignants font leurs études supérieures à la faculté de l'éducation, ce qui les conduit à ne pas négliger les références des informations trouvées. Ils respectent alors la citation des références.

\section{Conclusion}

L'étude actuelle s'inscrit dans le domaine des compétences informationnelles, domaine ayant son importance et sa pertinence scientifique et pratique, notamment dans le monde de l'information qui nous entoure. L'analyse des données recueillies lors de l'étude montre la présence de cinq domaines des compétences informationnelles: compétences générales, la reconnaissance du besoin informationnel, la recherche, l'évaluation et l'utilisation de l'information sur le Web. L'étude indique également le niveau moyen des enseignants du FLÉ en ce qui concerne la maîtrise de ces domaines. L'étude recommande alors d'intégrer les compétences informationnelles dans le programme de la formation académique et professionnelle de futurs enseignants aux facultés de l'éducation en général et de futurs enseignants du FLÉ en particulier. Ceci leur permet de confronter au monde de l'information qui les entoure, de bien faire leurs études universitaires, de mieux pratiquer leur métier et bien enseigner ces compétences à leurs élèves. L'étude recommande également de présenter des ateliers théoriques et pratiques aux enseignants - futurs et ce qui sont en poste - de la part des bibliothécaires de l'université dans différents thèmes, notamment la citation, le plagiat, les critères de l'évaluation et les droits de la propriété culturelle des auteurs.

Les résultats de l'étude contribuent à enrichir la connaissance dans le domaine des compétences des TIC, domaine primordial pour les apprenants, les enseignants et pour tous les individus de la société du savoir. L'étude introduit un cadre conceptuel qui permet aux futurs et 
actuels enseignants de comprendre la conception de la compétence, de l'information, des compétences informationnelles et de lire ce que la littérature présente dans ce domaine. L'étude identifie également certaines compétences informationnelles que les enseignants doivent avoir. Sur le plan pratique, l'étude attire l'attention des enseignants à évaluer les informations obtenues sur le Web, à distinguer entre le plagiat et la citation et à la nécessité d'éviter le plagiat lors de l'utilisation des informations numériques. L'étude a donc essayé d'enrichir ce domaine de recherche et de proposer certaines recommandations qui améliorent la formation des enseignants du FLÉ. Néanmoins, il serait pertinent de réaliser d'autres études auprès des étudiants universitaires afin d'obtenir un portrait détaillé concernant leur formation académique et pédagogique et pour examiner la présence/la maîtrise des compétences informationnelles chez eux. Il serait également important de mener d'autres études dans le domaine de l'évaluation des informations numériques, domaine assez différent de l'évaluation des informations trouvées en papier.

\section{Remerciement}

La chercheuse remercie tous les membres du jury et les enseignants participants à l'étude. Elle remercie également Dr. Sabry Basset à la faculté de l'éducation, Université de Sohag pour ses conseils et ses suggestions à améliorer la qualité du travail.

\section{Références}

Aillerie, K. (2012). Gestion de l'incertitude et compétence informationnelle : jalons pour approche comparative

des situations professionnelle et sociale. $4^{\mathrm{e}}$ édition du Colloque Spécialisé en Sciences de l'Information

(COSSI) «Information, incertitudes, intelligences ». Alvarez, J.-F. et Gisbert, M. (2015). Information literacy grade of secondary school teachers on Spain Beliefs

and self-perception. Comunicar, 23(45), 187-194. Repéré à http;//doi.org/10.3916/ C45-2015-20.

Andretta, S. (2007). Phenomenography: a conceptual framework for information literacy education. Aslib

Proceedings, 59(2), 152-168. 
Angeloro, R. (2010). Le profil Maître-TIC à la formation initiale des maîtres. Québec français, 159, 67-69.

April, J. et Beaudoin, M. (2006). Projet d'intégration des compétences informationnelles : mise à l'essai d'un

dispositif en enseignement préscolaire et primaire. Documentation et bibliothèques, 52(3), 173-181.

Archambault, C., Diane, B., Daphné, B. et Jean-Yves, C. (2009).

Comment évaluer la pertinence de

l'information et les sources d'information. Biblio Guide 17 : 1-2. Bibliothèque Myriam \& J.-Robert

Ouimet, HEC Montréal.

Arsac, J. (2003). Cité dans les «tablettes d'Ourouk, «Définition de l'information » $\mathrm{n}^{\circ} 2$, 2003. WWW.ourouk.fr.

Baschwitz, B. et Antonia, M. (2010). Comment me documenter?

Bruxelles : De Boeck.

Basque, J. (2005). Une réflexion sur les fonctions attribuées aux TIC en enseignement universitaire. Revue

internationale des technologies en pédagogie universitaire, 2(1), 30-41.

Bawden, D. (2001). The shifting terminoloogies of information. Aslib Proceedings, 53(3), 93-98.

Brich, A. et Irvine, V. (2009). Preservice teachers' acceptance of ICT integration in the classroom: Applying the

UTAUT model. Education Media International, 46(4), 295-315.

Chai, C. S. et Lim, C. P. (2011). The Internet and teacher education: Traversing between the digitized world and

schools. Internet and Higher Education, 14(1), 3-9.

Chevilotte, S. (2007). Maîtrise de l'information? Éducation à l'information? Culture informationnelle? Les

dossiers de l'Ingénierie Éducative, 57, 16-19.

Chevillotte, S. (2005). Bibliothèques et information literacy. Un état de 1'art. Bulletin des bibliothèques de

France, 50(2), 1-8.

Conférence des recteurs et des principaux des universités du Québec. (2005). Norme sur les compétences

informationnelles dans l'enseignement supérieur de l'Association of College \& Research Libraries

(ACRL). Montréal, QC : CREPUQ. Repéré à 
http://www.crepuq.qc.ca/IMG/pdf/normeacrl-Web-03-05-v4.pdf. Conseil de l'Europe. (2001). Cadre européen commun de référence pour les langues : apprendre, enseigner, évaluer. Paris: Didier.

Dohn, N. B. (2009). Web 2.0: Internet tensions and evident challenges for education. International Journal of

Computer-Supported Collaborative Learning, 4(3), 343-363.

Drent, M. et Meelissen, M. (2008). Which factors obstruct or stimulate teacher educators to use ICT

innovatively? Computers and Education, 51(1), 187-199.

Dumouchel, G. (2016). Les compétences informationnelles des futurs enseignants québécois sur le Web (Thèse

de doctorat). Université de Montréal.

FONDAR. (2004). Pour une approche intégrée d'apprentissage continu des compétences informationnelles.

Université de Québec.

Fox, B. (2007). Teaching through technology. Changing practices in two universities. International Journal on

E-Learning, 6(2), 187-203.

Galanouli, D. et Mcnair, V. (2001). Students' perception of ICT-related support in teaching placements. Journal

of Computer Assisted learning, 17(4), 396-408.

Gauthier, R. (2007). Une démarche inductive, un choix qui s'impose dans les études sur le sens de l'expérience

scolaire : l'exemple d'une recherche portant sur le rapport à l'institution scolaire en milieu autochtone.

Recherches qualitatives, 27(2), 78-103.

Gervais, S. (2004). Les habiletés en recherche d'information des étudiant(e)s universitaires : une observation

(Mémoire de maîtrise). Université de Montréal.

Gervais, S. et Clément, A. (2005). Habilités en recherche d'information des étudiants de première année

universitaire en sciences de l'éducation. Documentation et Bibliothèques, 51(4), 241-260.

Giroux, P., Gagnon, M., Gremion, C. et Heinzen, S. (2014). Regard de futurs enseignants sur l'importance des

compétences TIC (Internet) pour les jeunes et la responsabilité de divers intervenants à cet égard. 
Formation et profession, 22(1), 13-26.

Jonnaert, P., Barrette, J., Boufrahi, S. et Masciotra, D. (2004). Contribution critique au développement

des programmes d'études: compétences, constructivisme et interdisciplinarité. Revue des sciences

de l'éducation, 30(3), 667-696.

Kaplan, A. M. et Haenlein, M. (2010). Users of the world, unite! The challenges and opportunities of social

media. Business Horizons, 53(1), 59-68.

Karsenti, T. (2015). Les devoirs: ce que dit la recherche, stratégies gagnantes, impact des technologies.

Montréal : Grand Doc.

Karsenti T. et Collin, S. (2013). TIC et éducation : avantages, défis et perspectives futures. Éducation

et francophonie, XLI, 1-6.

Kuhlthau, C.C. (2004). Seeking meaning: A process approach to library and information services $(2 \mathrm{e}$

éd.). Westport, CT: Libraries Unlimited.

Kuhlthau, C.C. (1993). Seeking meaning: A process approach to library and information services.

Norwood. NJ: Ablex.

L'association des directeurs \& personnels de direction des bibliothèques universitaires et de la documentation.

(2012). Référentiel de compétences informationnelles. Document pdf.

Legendre, R. (2005). Dictionnaire actuel de l'éducation. Montréal, QC :Guérin.

Le Petit Larousse (1995). Dictionnaire grand format. Paris.

Le Robert. (1995). Dictionnaire le robert, collection les usuels. Paris.

Loisy, C. (2007). Le C2i2e : un référentiel de compétences dans une formation professionnelle. Actes du

colloque «Compétences, emploi et enseignement supérieur. Fondements scientifiques, développements,

attentes sociétales. » Universités de Bretagne.

Martinet, M., Raymond, D. et Gauthier, C. (2001). La formation à l'enseignement : les orientations, les

compétences professionnelles. Québec, Canada: Ministère de l'Éducation du Québec. 
Miles, M. B. et Huberman, M. (2003). Analyse des données qualitatives. Paris : de Boeck.

Ministère de l'éducation nationale, de l'enseignement supérieur et de la recherche. (2013). Référentiel des

compétences professionnelles des métiers du professorat et de l'éducation. Journal Officiel de la

République Français.

Mittermeyer, D. et Quirion, D. (2003). Étude sur les connaissances en recherche documentaire des étudiants

entrant au ler cycle dans les universités québécoises. Montréal:

\section{CREPUQ.}

Nel, C. Big6 process model. Repéré à

http://nmasse.com/courses/ref/big6/big6.htm, consulté le 17

novembre, 2018.

OCDE. (2009). 21st century skills and competences for new millennium learners in OECD countries (EDU

Working paper no.41). Repéré à https://www.oecd-

ilibrary.org/education/21st-century-skills-and-

competences-for-new-millennium-learners-in-oecd-

countries_218525261154

OCDE. (1996). Évaluer et réformer les systèmes éducatifs. Paris : OCDE.

Othman, D. D. (2008). Coming terms with Web 2.0. Reference Reviews, 21(7), 5-6.

Passerieux et Verreault. (2013). Évaluer et citer ses sources. InfoSphère : Sciences humaines et sciences sociales.

Service des bibliothèques de l'UQAM.

Pinte, J.-P. (2005). La relation au savoir et les outils de la veille pédagogique. Pédagogie collégiale, 18(3), 23-

30.

Pochet, B. (2012). Lire et écrire la littérature scientifique. Presses agronomiques de Gembloux.

Pomagni, P. et Wild, V. (1998). L'intelligence économique au service de l'entreprise. Paris : Les Presses du

Management.

Potvin, V. (2013). Évaluation critique des sources d'information en ligne chez les adolescents du secondaire : 
effets d'interventions pédagogiques dans le cours monde contemporain (Mémoire de maîtrise). Université de Montréal.

Puren, C. (2008). Intervention Rencontres FLE de Barcelone, 14 novembre, 2008.

Rasmy, A. (2010). Méthodologie de construction d'un instrument de mesure des compétences technologiques et

informationnelles des étudiants de l'enseignement supérieur.

Montréal, QC : Université de Montréal.

Redecker, C. (2009). Review of Learning 2.0 Practices: Study on the Impact of Web 2.0 Innovations on

Education and Training in Europe. Séville, Espagne: Institute for Prospective technological Studies.

Robert, A.D. et Bouillaguet, A. (1997). L'analyse de contenu. (Coll. « Que sais-je? »). Paris : PUF.

Sacchanand, C. (2011). Concevoir des stratégies pour développer les compétences informationnelles des élèves

thaïlandais. World Library and Information Congress: 77th IFLA General Conference and Assembly. En

ligne: http://confernce.ifla.org/ifla77

Sahut, G. (2017). L'enseignement de l'évaluation critique de l'information numérique. Tic\&société, 11(1), 223-

248. En ligne http://journals.openedition.org/ticetsciete/2321.

Salem, H.T.A. (2013). Une cyberenquête proposée pour développer les compétences de l'expose oral chez les

étudiants de la faculté de la Pédagogie de Sohag. Journal of Arabic Studies in Education \& Psychology,

44(3), 1-62.

Salem, H.T.A. (2012). Effet de l'apprentissage de l'écoute via des sites Web sur la motivation des apprenants de

FLE à la faculté de Pédagogie de Sohag. Revue de la faculté de Pédagogie de Sohag, 32, 1-46.

Salem, H.T.A. (2010). Impact de l'usage d'Internet sur le développement de la compréhension orale chez les

étudiants de FLE à la faculté de Pédagogie de Sohag. Études en curricula et méthodes d'enseignement,

171, 23-56. 
Salem, H.T.A. (2009). L'effet du multimédia sur le développement de la compréhension écrite chez les étudiants

de la faculté de Pédagogie de Sohag. Revue de la faculté de Pédagogie de Sohag, 26, 1-29.

Savoie-Zjac, L. (2009). L'entrevue-semi-dirigée. Dans B. Gautier (dir.), Recherche sociale : de la problématique

à la collecte des données (5 ${ }^{\mathrm{e}}$ éd., p. 337-360). Québec, QC :

Presses de l'Université du Québec.

Soung, S. (2017). Critères d'évaluation de l'information scientifique à l'ère numérique Cas des étudiants aux

cycles supérieurs en éducation des universités québécoises.

Documentation et bibliothèques, 63(3), 36-49.

Tardif, J. (2001). L'évaluation des compétences: documenter le parcours de développement. Montréal, QC :

Chenelière Éducation.

Teo, T., Chai, C. S., Hung, D. et Lee, C. B. (2008). Beliefs about teaching and uses of technology among pre-

service teachers. Asia Pacific Journal of Teacher Education, 36(2), 163-174.

Underwood, J. et Dillon, G. (2011). Chasing dreams and recognising realities: Teachers'responses to ICT.

Technology, Pedagogy and Education, 20(3), 317-330.

UNESCO. (2006). Programme information pour tous. France: Paris, UNESCO. Repéré à

https://unesdoc.unesco.org/images/0015/001502/150279f.pdf.

UNESCO. (2005). Vers les sociétés du savoir. France : Paris, UNESCO. Repéré à

https://unesdoc.unesco.org/images/0014/001419/141907f.pdf.

Villeneuve, S., Collin, S. et Karsenti, T. (2013). Facteurs influençant l'utilisation des technologies de

l'information et de la communication chez les stagiaires en enseignement du secondaire. Education et

francophonie, XLI(1), 30-44.

Waguih, M. (2002). L'efficacité de l'emploi de multimédia au développement des compétences du professeur en

enseignant l'expression en français selon l'approche écrite communicative (Mémoire de maîtrise).

Université d'Ain Chams. 
Les compétences informationnelles nécessaires à la formation contenue $\ldots \ldots \ldots \ldots \ldots \ldots$

Walha, L., et Peters, M. (2011). Les compétences technopédagogiques des enseignants et les obstacles à

l'intégration des TIC. En ligne :

https://www.epi.asso.fr/revue/articles/a1106f.htm

Annexe 1

Liste des membres du jury qui ont évalué les outils de l'étude

\begin{tabular}{|c|c|c|}
\hline No & Le nom & La profession \\
\hline 1 & $\begin{array}{l}\text { Dr. Isabelle M- } \\
\text { G. }\end{array}$ & $\begin{array}{l}\text { Professeure titulaire, faculté des sciences de } \\
\text { l'éducation, UdeM }\end{array}$ \\
\hline 2 & $\begin{array}{l}\text { Dr. Annie } \\
\text { Charron }\end{array}$ & $\begin{array}{l}\text { Professeure, faculté des sciences de l'éducation, } \\
\text { Université d'UQÀM }\end{array}$ \\
\hline 3 & $\begin{array}{l}\text { Dr. Marcel } \\
\text { Thouin }\end{array}$ & $\begin{array}{l}\text { Professeur titulaire, faculté des sciences de } \\
\text { l'éducation, UdeM }\end{array}$ \\
\hline 4 & $\begin{array}{l}\text { Dr. Karima } \\
\text { kafif }\end{array}$ & $\begin{array}{l}\text { PH.D, UdeM, bibliothécaire à la bibliothèque de } \\
\text { l'université d'Ottawa }\end{array}$ \\
\hline 5 & $\begin{array}{l}\text { Dr. Rania } \\
\text { Mohamad }\end{array}$ & $\begin{array}{l}\text { Maitre de conférences, faculté de l'éducation, } \\
\text { université de Demiatte }\end{array}$ \\
\hline 6 & $\begin{array}{l}\text { Dr. Randa } \\
\text { Essam }\end{array}$ & $\begin{array}{l}\text { Maitre de conférences, faculté de l'éducation, } \\
\text { université d'Assuitte }\end{array}$ \\
\hline 7 & Asmaa Hanafy & $\begin{array}{l}\text { Enseignante à l'institut de jeunes filles d'Al- } \\
\text { Azhar de Sohag }\end{array}$ \\
\hline 8 & $\begin{array}{l}\text { Tamer El- } \\
\text { Refaye }\end{array}$ & $\begin{array}{l}\text { Enseignant à l'institut secondaire des garçons, } \\
\text { Sohag }\end{array}$ \\
\hline 9 & Youssra Adli & $\begin{array}{l}\text { Enseignante à l'école secondaire d'El Dawa El } \\
\text { Ismail }\end{array}$ \\
\hline 10 & $\begin{array}{l}\text { Nessrin } \\
\text { Mahmoud }\end{array}$ & $\begin{array}{l}\text { Enseignante à l'institut de jeunes filles d'Al- } \\
\text { Azhar de Sohag }\end{array}$ \\
\hline 11 & $\begin{array}{l}\text { Mohamed } \\
\text { Samir }\end{array}$ & $\begin{array}{l}\text { Enseignant à l'école d'El-Shayna, Naag } \\
\text { Hamaady }\end{array}$ \\
\hline 12 & Samah Hany & $\begin{array}{l}\text { Enseignante, l'école préparatoire ancienne des } \\
\text { garçons, Sohag }\end{array}$ \\
\hline
\end{tabular}


Annexe 2

Liste des enseignants participants à l'étude

\begin{tabular}{|c|c|c|c|}
\hline Le nom & L'école & Le nom & L'école \\
\hline 1.Asmaa Hanafy & $\begin{array}{l}\text { L'institut des filles d'Al- } \\
\text { Azhar, Sohag }\end{array}$ & $\begin{array}{l}\text { 24.Fatema } \\
\text { Mohamoud }\end{array}$ & $\begin{array}{l}\text { L'école El- } \\
\text { Zuhoor, Sohag }\end{array}$ \\
\hline 2. Samah Hany & $\begin{array}{l}\text { L'école préparatoire } \\
\text { ancienne des garçons, } \\
\text { Sohag }\end{array}$ & $\begin{array}{l}\text { 25. Nahed } \\
\text { Yassin }\end{array}$ & $\begin{array}{l}\text { L'école } \\
\text { préparatoire } \\
\text { mixte, El- } \\
\text { Hawawesh }\end{array}$ \\
\hline 3.Tamer El-Refaye & $\begin{array}{l}\text { L'institut secondaire des } \\
\text { garçons, Sohag }\end{array}$ & $\begin{array}{l}\text { 26. Azaa El- } \\
\text { Sharquawi }\end{array}$ & $\begin{array}{l}\text { L'école } \\
\text { préparatoire d'El } \\
\text { Sadat, Akmim }\end{array}$ \\
\hline 4. Youssra Adli & $\begin{array}{l}\text { L'école secondaire des } \\
\text { filles El Dawa El Ismail }\end{array}$ & $\begin{array}{l}\text { 27. Amal } \\
\text { Abdelfatah }\end{array}$ & $\begin{array}{l}\text { L'école } \\
\text { préparatoire d'El } \\
\text { Sadat, Akmim }\end{array}$ \\
\hline 5.Nessrin Mahmoud & $\begin{array}{l}\text { L'institut des filles d'Al- } \\
\text { Azhar, Sohag }\end{array}$ & $\begin{array}{l}\text { 28. Manal } \\
\text { Shaker }\end{array}$ & $\begin{array}{l}\text { L'école } \\
\text { préparatoire } \\
\text { mixte, El- } \\
\text { Hawawesh }\end{array}$ \\
\hline 6. Mohamed Samir & $\begin{array}{l}\text { L'école d'El-Shayna, } \\
\text { Naag Hamaady }\end{array}$ & $\begin{array}{l}\text { 29.Marwa } \\
\text { Fathouh }\end{array}$ & $\begin{array}{l}\text { L'institut } \\
\text { secondaire des } \\
\text { filles d'El Salah }\end{array}$ \\
\hline 7. Hoda Mustafa & $\begin{array}{l}\text { L'école préparatoire } \\
\text { d'Ahmed Abdelraouf, } \\
\text { Tahta }\end{array}$ & $\begin{array}{l}\text { 30.Noha } \\
\text { Mohamad }\end{array}$ & $\begin{array}{l}\text { L'institut } \\
\text { secondaire des } \\
\text { filles d'El Salah }\end{array}$ \\
\hline 8. Ahmed Saber & $\begin{array}{l}\text { L'école préparatoire des } \\
\text { garçons, Akmim }\end{array}$ & $\begin{array}{l}\text { 31.Hatem Salah- } \\
\text { eldin }\end{array}$ & $\begin{array}{l}\text { L'institut } \\
\text { préparatoire et } \\
\text { secondaire des } \\
\text { filles, Balasfora }\end{array}$ \\
\hline 9. Esslam Mohamed & $\begin{array}{l}\text { L'école préparatoire des } \\
\text { filles, Akmim, }\end{array}$ & 32. Eman Sabry & $\begin{array}{l}\text { L'institut } \\
\text { préparatoire et } \\
\text { secondaire des } \\
\text { filles, El-Kawther }\end{array}$ \\
\hline 10. Samwael Habib & $\begin{array}{l}\text { L'école d'El-Salam, El- } \\
\text { Kawther }\end{array}$ & $\begin{array}{l}\text { 33. Mustafa } \\
\text { Hamed }\end{array}$ & $\begin{array}{l}\text { L'école } \\
\text { secondaire mixte } \\
\text { d'El Salah }\end{array}$ \\
\hline 11. Mustafa Hesham & $\begin{array}{l}\text { L'école d'El-Salam, El- } \\
\text { Kawther }\end{array}$ & $\begin{array}{l}\text { 34. Ashraf } \\
\text { Ayman }\end{array}$ & $\begin{array}{l}\text { L'école } \\
\text { secondaire mixte } \\
\text { d'El Salah }\end{array}$ \\
\hline 12. Wessam Mahmoud & $\begin{array}{l}\text { L'école secondaire des } \\
\text { filles, Quina }\end{array}$ & $\begin{array}{l}\text { 35. Osama El- } \\
\text { Saed }\end{array}$ & $\begin{array}{l}\text { L'école } \\
\text { secondaire mixte } \\
\text { d'El Salah }\end{array}$ \\
\hline 13. Essraa Ali & $\begin{array}{l}\text { L'école du secondaire des } \\
\text { filles, Quina }\end{array}$ & $\begin{array}{l}\text { 36. Hussein } \\
\text { Ahmed }\end{array}$ & $\begin{array}{l}\text { L'institut } \\
\text { secondaire des } \\
\text { garçons de Mashta }\end{array}$ \\
\hline
\end{tabular}


Les compétences informationnelles nécessaires à la formation contenue $\ldots \ldots \ldots \ldots \ldots \ldots$

\begin{tabular}{|c|c|c|c|}
\hline 14.Amira Mustafa & $\begin{array}{l}\text { L'école secondaire des } \\
\text { filles, Quina }\end{array}$ & $\begin{array}{l}\text { 37. Omar } \\
\text { Ahmed }\end{array}$ & $\begin{array}{l}\text { L'institut } \\
\text { secondaire des } \\
\text { garçons d'El- } \\
\text { Atamna }\end{array}$ \\
\hline 15.Yasser Ossman & $\begin{array}{l}\text { L'école préparatoire des } \\
\text { garçons, Quina }\end{array}$ & $\begin{array}{l}\text { 38. Ashraf } \\
\text { Mohamed }\end{array}$ & $\begin{array}{l}\text { L'institut } \\
\text { secondaire des } \\
\text { garçons de Tema }\end{array}$ \\
\hline 16.Saleh Emara & $\begin{array}{l}\text { L'institut des garçons } \\
\text { d'Al-Azhar }\end{array}$ & $\begin{array}{l}\text { 39. Mahmoud } \\
\text { Kamal }\end{array}$ & $\begin{array}{l}\text { L'institut } \\
\text { secondaire des } \\
\text { garçons de Tema }\end{array}$ \\
\hline 17.Hussam Ali & $\begin{array}{l}\text { L'école préparatoire } \\
\text { mixte, Assuitte }\end{array}$ & 40.Khlaed Zaki & $\begin{array}{l}\text { L'école } \\
\text { secondaire El- } \\
\text { Shaheed Khayrat, } \\
\text { Naag Hamaady }\end{array}$ \\
\hline 18.Saad El-din Alam & $\begin{array}{l}\text { L'école secondaire mixte, } \\
\text { Assuitte }\end{array}$ & $\begin{array}{l}\text { 41. Mohamed } \\
\text { Ebrahim }\end{array}$ & $\begin{array}{l}\text { L'école } \\
\text { secondaire des } \\
\text { filles, Naag } \\
\text { Hamaady }\end{array}$ \\
\hline 19.Mohamed Karmoty & $\begin{array}{l}\text { L'école secondaire mixte, } \\
\text { Assuitte }\end{array}$ & $\begin{array}{l}\text { 42. Mohamed } \\
\text { Hassan }\end{array}$ & $\begin{array}{l}\text { L'école } \\
\text { secondaire des } \\
\text { filles, Naag } \\
\text { Hamaady }\end{array}$ \\
\hline 20.Sherin Galal & $\begin{array}{l}\text { L'école secondaire des } \\
\text { filles El Dawa El Ismail }\end{array}$ & $\begin{array}{l}\text { 43. Zeinab } \\
\text { Mohamed }\end{array}$ & $\begin{array}{l}\text { L'école d'El- } \\
\text { Shayna, Naag } \\
\text { Hamaady }\end{array}$ \\
\hline 21.Sumiya Wahby & $\begin{array}{l}\text { L'école secondaire des } \\
\text { filles, El-Hawawesh }\end{array}$ & 44.Ali Salah & $\begin{array}{l}\text { L'école d'El- } \\
\text { Sadat, Naag } \\
\text { Hamaady }\end{array}$ \\
\hline 22.Eman Mahmoud & $\begin{array}{l}\text { L'école secondaire des } \\
\text { filles El Dawa El Ismail }\end{array}$ & $\begin{array}{l}\text { 45.Asmaa } \\
\text { Abdelrasul }\end{array}$ & $\begin{array}{l}\text { L'école } \\
\text { secondaire des } \\
\text { garçons, El- } \\
\text { Hawawesh }\end{array}$ \\
\hline 23.Refaat Mohamad & $\begin{array}{l}\text { Directeur : Ahkmim } \\
\text { administration, Sohag }\end{array}$ & & \\
\hline
\end{tabular}

